\title{
Analysis of Potential Hub Genes and Related Transcription Factors in Rosacea Patients using Bioinformatics Analysis
}

\section{Xin Wang}

Peking Union Medical College Plastic Hospital

\section{Wenfang Dong}

Chinese Academy of Medical Sciences \& Peking Union Medical College Plastic Surgery Hospital and Institute

\section{Huan Wang}

Peking Union Medical College Plastic Hospital

\section{Jianjun You}

Peking Union Medical College Plastic Hospital

\section{Ruobing Zheng}

Chinese Academy of Medical Sciences \& Peking Union Medical College Plastic Surgery Hospital and Institute

\section{Yihao Xu}

Peking Union Medical College Plastic Hospital

Fei Fan ( $\nabla$ fanfei@psh.pumc.edu.cn )

Chinese Academy of Medical Sciences \& Peking Union Medical College Plastic Surgery Hospital and Institute

\section{Research}

Keywords: Rosacea, Diferentially expressed genes, hub gene, bioinformatics, Transcriptional factors

Posted Date: August 30th, 2021

DOI: https://doi.org/10.21203/rs.3.rs-824062/v1

License: (a) (i) This work is licensed under a Creative Commons Attribution 4.0 International License. Read Full License 


\section{Abstract}

Objective The aim of this study is to discover the adipocyte genes and pathways involved in rosacea using bioinformatics analysis.

Methods The GSE65914 gene expression profile was obtained. The GEO2R tool was used to screen out differentially expressed genes (DEGs). It was further analyzed with Gene Ontology (GO) to explore functional annotation and Kyoto Encyclopedia of Genes and Genomes (KEGG) to explore cell signaling pathways. Protein-protein interaction (PPI) networks among the DEGs were found by STRING databases and visualized in Cytoscape software. The related transcription factors regulatory network of the DEGs were also constructed.

Results A total of 254 DEGs, including 72 up-regulated genes and 182 down-regulated genes, were obtained in rosacea samples. The biological functions of DEGs are mainly involved in the inflammatory response and chemokine activity. A PPI network consisting of 217 nodes and 710 edges was constructed using STRING, and ten hub genes were identified with Cytoscape software. Some transcriptional factors were also found to interact with these hub DEGs.

Conclusion In this study, we obtained ten hub genes, including CXCL8, CCR5, CXCR4, CXCL10, MMP9, CD2, CCL19, CXCL9, CCL5, CD3D, which play an essential role in the pathology of rosacea, and these genes may provide a basis for the screening of treatment biomarkers for rosacea in the future.

\section{Introduction}

Rosacea is a chronic facial inflammatory disease characterized by transient erythema or persistent erythema, papules, pustules, or telangiectasia in the central face (cheeks, chin, nose, and central part of forehead), and some patients are accompanied by acute symptoms such as burning and itching[1, 2]. It is divided into four main subtypes, including erythemato-telangiectatic (ETR), papulopustular rosacea (PPR), phymatous rosacea (PHR), and ocular rosacea (OR), which ETR is the most common type[3, 4]. It is estimated that the incidence rate is $5.46 \%$ worldwide, and it is more frequent among females between 45 and 60 years of age[4]. In recent years, there is more and more skin barrier damage caused by abuse and improper treatment of skincare products, and the proportion of rosacea in young groups is also gradually increasing. Although rosacea is not life-threatening, it can have a negative impact on the patient's quality of life. Moreover, the related comorbidities, including cardiovascular disease, malignancy, depression, and generalized anxiety disorder, have been identified in rosacea patients[5, 6]. The mechanisms of rosacea are not fully understood, but vascular, immune, and neurological dysfunctions seem to play an essential role in the process[3]. Research suggests that modification in multiple genes and signaling pathways are associated with controlling the development of rosacea. However, a lack of investigation on the precise molecular mechanisms of rosacea development limits the treatment efficacy of the disease. 
Previous studies showed the cytokines released by inflammatory cells such as leukocytes and macrophages after environmental triggers, such as heat, spicy food, UV, physical or chemical stimuli, and microbes. For example, the studies suggested that the Th17 pathway is activated in rosacea after trigger factors, and IL-17, one of the Th17 signature cytokines, can stimulate CXCL8 and CXCL5 expression, which can promote angiogenesis. It can also stimulate MMP-9 expression to active LL-37, which induces cytokine production, chemotaxis, and angiogenesis in rosacea $[7,8,9,10]$. The mammalian target of rapamycin (mTOR) plays an irreplaceable role in regulating nutrients and energy status, cellular stressors, and cytokines[11]. It has been reported in the literature that hyperactivated mTORC1 signaling is significantly associated with rosacea patients by binding to Toll-like receptor 2 (TLR2) with the regulation of immune responses and inflammatory responses $[12,13]$. Therefore, we have reason to speculate that there should be many potential genes in the pathological process of rosacea that may play important regulatory roles.

With the development of genomics technology, there are large amounts of data in the field of rosacea. To detect the differentially expressed genes (DEGs) between rosacea and healthy human tissue, bioinformatics methods were used to analyze the gene expression profiling data downloaded from the Gene Expression Omnibus (GEO) database to screen for hub genes through the PPI network and subsequently analyze their functions.

\section{Results}

\section{Identification of DEGs}

The gene expression profile of the GSE65914 dataset was uploaded to the GEO2R to screen DEGs. The result of the normalization of data among the arrays of the series matrix files of the GSE65914 dataset is shown in Fig. S1. Based on the criteria of $P<0.05$ and $|\log F C| \geq 2$, a total of $254 \mathrm{DEG}$ were identified from GSE65914, including 72 up-regulated genes and 182 downregulated genes. Volcano plots and heat maps for the distribution of DEGs were generated using Prism 8.0 software (Figures. 1 and 2).

\section{Functional enrichment analyses of DEGs}

GO function (including biological process, molecular function, and cellular component) and KEGG pathway enrichment analysis for DEGs was performed using a functional annotation tool of DAVID. The results were summarized in Table 1. On GO analysis, inflammatory response process (GO:0006954; FDR $=2.22 \mathrm{E}-13)$, cell-cell signaling 
Table 1

Significantly enriched GO terms and KEGG pathways of DEGs

\begin{tabular}{|c|c|c|c|c|}
\hline Pathway ID & Description & Count & P Value & FDR \\
\hline \multicolumn{5}{|l|}{ BP } \\
\hline GO:0006954 & inflammatory response & 31 & $1.90 \mathrm{E}-16$ & $2.22 \mathrm{E}-13$ \\
\hline GO:0005615 & extracellular space & 54 & $2.37 \mathrm{E}-15$ & $4.03 \mathrm{E}-13$ \\
\hline GO:0005576 & extracellular region & 57 & $6.08 \mathrm{E}-14$ & 5.17E-12 \\
\hline GO:0006955 & immune response & 28 & $1.22 \mathrm{E}-12$ & $7.16 \mathrm{E}-10$ \\
\hline G0:0008009 & chemokine activity & 10 & 5.47E-09 & $2.06 \mathrm{E}-06$ \\
\hline G0:0070098 & chemokine-mediated signaling pathway & 11 & $1.24 \mathrm{E}-08$ & $4.84 \mathrm{E}-06$ \\
\hline G0:0004252 & serine-type endopeptidase activity & 16 & 4.19E-07 & 7.88E-05 \\
\hline G0:0007267 & cell-cell signaling & 16 & $4.52 \mathrm{E}-07$ & $1.32 \mathrm{E}-04$ \\
\hline \multicolumn{5}{|l|}{$\mathrm{CC}$} \\
\hline GO:0070062 & extracellular exosome & 61 & $1.61 \mathrm{E}-06$ & $9.12 \mathrm{E}-05$ \\
\hline GO:0006935 & chemotaxis & 11 & 2.17E-06 & $4.24 \mathrm{E}-04$ \\
\hline GO:0005198 & structural molecule activity & 14 & $8.55 \mathrm{E}-06$ & 0.001072 \\
\hline G0:0045087 & innate immune response & 18 & $1.82 \mathrm{E}-05$ & 0.002371 \\
\hline \multicolumn{5}{|l|}{ MF } \\
\hline G0:0006508 & proteolysis & 18 & $1.19 \mathrm{E}-04$ & 0.009934 \\
\hline G0:0009897 & external side of plasma membrane & 11 & $2.18 \mathrm{E}-04$ & 0.007421 \\
\hline \multicolumn{5}{|l|}{ KEGG } \\
\hline hsa04060 & Cytokine-cytokine receptor interaction & 15 & $3.49 \mathrm{E}-06$ & $4.26 \mathrm{E}-04$ \\
\hline hsa04062 & Chemokine signaling pathway & 13 & $5.87 \mathrm{E}-06$ & 4.26E-04 \\
\hline
\end{tabular}

process (GO:0007267; FDR $=1.32 \mathrm{E}-04)$, innate immune response process $(\mathrm{GO}: 0045087 ; \mathrm{FDR}=0.002371)$ were selected as the most significant pathway in each subontologies, respectively. KEGG pathway enrichment analysis result showed that cytokine-cytokine receptor interaction process $(p=3.49 E-06$, FDR $=4.26 \mathrm{E}-04$ ) was the top enriched pathway according to their p-values.

\section{PPI network construction and hub gene identification}

To further understand the correlation of DEGs and screen out hub genes, we constructed a PPI network based on the STRING online database. A total of 217 nodes and 710 edges were involved in the PPI network. Figure 3 presents the visualization of the network generated using Cytoscape software. Figure 4 
represents the ten selected hub genes with the highest degrees of connectivity selected as the hub genes. The results showed that all the hub genes were up-regulated genes. CXCL8 was the most outstanding gene with a connectivity degree of 18 , followed by CCR 5 with a connectivity degree of 15 . The subsequent GO function annotation analysis and KEGG pathway analysis results for hub genes are summarized in Table 2.

Table 2

GO and pathway enrich analysis of hub genes.

\begin{tabular}{|llccc|}
\hline Pathway ID & Description & Count & P Value & FDR \\
\hline BP & & & & \\
\hline G0:0070098 & Chemokine-mediated signaling pathway & 7 & $3.83 \mathrm{E}-13$ & $6.78 \mathrm{E}-11$ \\
\hline GO:0006935 & Chemotaxis & 6 & $2.29 \mathrm{E}-09$ & $2.03 \mathrm{E}-07$ \\
\hline GO:0006954 & Extracellular region & 7 & $1.01 \mathrm{E}-08$ & $5.95 \mathrm{E}-07$ \\
\hline GO:0006955 & Immune response & 6 & $1.12 \mathrm{E}-06$ & $3.97 \mathrm{E}-05$ \\
\hline GO:0007186 & G-protein coupled receptor signaling pathway & 10 & $1.69 \mathrm{E}-06$ & $4.99 \mathrm{E}-05$ \\
\hline CC & & & & \\
\hline GO:0005576 & Extracellular region & 7 & $3.13 \mathrm{E}-05$ & $7.51 \mathrm{E}-04$ \\
\hline GO:0005615 & Extracellular space & 6 & $2.14 \mathrm{E}-04$ & 0.001715 \\
\hline MF & & & & \\
\hline GO:0008009 & Chemokine activity & 5 & $7.81 \mathrm{E}-09$ & $3.67 \mathrm{E}-07$ \\
\hline KEGG & & & & \\
\hline hsa04062 & Chemokine signaling pathway & 7 & $2.83 \mathrm{E}-08$ & $1.05 \mathrm{E}-06$ \\
\hline hsa04060 & Cytokine-cytokine receptor interaction & 7 & $1.40 \mathrm{E}-07$ & $2.60 \mathrm{E}-06$ \\
\hline
\end{tabular}

\section{TF regulatory network analysis of hub genes}

We constructed a gene-TF regulatory network for these identified hub genes, including 56 interaction pairs among eight genes and 50 TFs (Fig. 5). MMP9 was found the most outstanding gene regulated by 22 TFs, followed by the CXCR4 gene regulated by 18 TFs. In addition, several TFs were found to regulate more than one hub gene, and 5 TFs were found with a connectivity degree $\geq 2$ in the gene-TF regulatory network, which shows that these TFs may have close interactions with these genes (Table 3 ). 
Table 3

The potential TFs of hub genes.

\begin{tabular}{|lll|}
\hline TFs & Genes & Count \\
\hline EZH2 & MMP9, CXCR4 & 2 \\
\hline CBF8 & CCR5, CXCR4 & 2 \\
\hline SUPTSH & CD2, CXCR4 & 2 \\
\hline WRNIP1 & CCL5, CXCR4 & 2 \\
\hline MXD4 & MMP9, CCL19 & 2 \\
\hline
\end{tabular}

\section{Discussion}

The present study uses the GEO database to obtain gene expression profiles from patients with rosacea and screens for DEGs. According to the criterion for DEGs (adjusted $p$-value $<0.05$ and $|\log F C| \geq 2$ ), we finally got 254 DEGs including 72 up-regulated genes and 182 down-regulated genes. These DEGs were associated with the GO BP terms such as inflammatory response, extracellular space, extracellular region, immune response. KEGG analysis showed the meaningful enrichment of pathways involved in cytokinecytokine receptor interaction and chemokine signaling pathway. Next, PPI analyses were carried out to identify the hub genes that may play a crucial regulatory role in the pathophysiology of rosacea. The hub genes included CXCL8, CCR5, CXCR4, CXCL10, MMP9, CD2, CCL19, CXCL9, CCL5, CD3D, and they are all up-regulated genes. Finally, we found some TFs, the main regulators of gene expression, with close interactions with hub DEGs.

C-X-C Motif Chemokine Ligand 8, 9, and 10 (CXCL-4,8,9,10) is a protein-coding gene. The protein encoded by these genes is a member of the $\mathrm{CXC}$ chemokine family and is a significant mediator of the inflammatory response. Some research has demonstrated that CXCL8 is involved in Melanoma and Bronchiolitis, and its encoded protein is commonly referred to as interleukin-8 (IL-8), a chemotactic factor that attracts neutrophils, basophils, and T-cells[14]. Previous studies suggested that in rosacea patients, IL-17 can stimulate CXCL8 and CXCL5 expression, promoting angiogenesis, and an IL-17 inhibitor, secukinumab, was tested as an agent in patients with papulopustular rosacea[7, 8, 9]. In addition, proinflammatory cytokines encoded by CXCL10 are involved in various processes, such as chemotaxis, differentiation, and activation of peripheral immune cells, regulation of cell growth, apoptosis, and regulation of vascular inhibition[15, 16]. The related pathway was CCR5 Pathway in Macrophages, the same as the CCR5 gene, which played a role in controlling granulocytic lineage proliferation or differentiation[17]. Thus, CXCL and CCR5 may play a vital role in the occurrence and development of rosacea.

Matrix metalloproteases (matrix metalloproteinase, MMPs), also called matrixes, play an essential role in local proteolysis of the extracellular matrix and leukocyte migration[18, 19]. Tuğba Falay Gur et al. found that the serum levels of MMP9 were higher in rosacea patients[20]. Moreover, Woo YR et al. suggested 
that MMP-9 expression can activate LL-37, inducing cytokine production and angiogenesis in rosacea patients[10]. CD2, CCL19, CCL5, and CD3D have not yet been reported in cases of rosacea. But, on review of the literature, we found that these genes are associated with inflammatory and immunological responses. They may use active several chemokine receptors, not only for T-cells and B-cells but eosinophils, which are pathologically similar to rosacea. Therefore, these hub genes feature as potential prognostic or diagnostic markers, as well as potential therapeutic targets.

TFs, the primary regulators of gene expression, are related to the pathogenic processes of rosacea. Our study also found some TFs with a close relationship with hub DEGs. CBFB is a transcription factor that is essential for hematopoiesis and osteogenesis. Mutations of CBFB can lead to estrogen receptor-positive breast cancer, acute Myeloid Leukemia[21, 22]. WRNIP1 functions as a modulator of initiation during the DNA synthesis process, which its mutation can cause Werner's syndrome, a rare autosomal recessive disorder[23]. In addition, EZH2 may play a role in the hematopoietic and central nervous systems, and its dysregulation may cause the development of cancer[24]. TFs constructed a network with hub DEGs, thus showing that these TF activities' changes may result in pathogenic expression and function of hub DEGs of rosacea.

However, our study had several limitations. First, the sample size was limited, and we only analyzed a dataset. Moreover, the mechanisms of some hub genes remain unclear in the pathological process.

\section{Conclusion}

Our bioinformatics analysis identified 254 DEGs between rosacea patients and normal tissues based on the GEO database. Among them, ten hub genes might be the core genes of rosacea, including CXCL8, CCR5, CXCR4, CXCL10, MMP9, CD2, CCL19, CXCL9, CCL5, CD3D. All of them were up-regulated in rosacea, and TFs were also found with these hub DEGs from the gene-TF network. These results may provide a basis for the screening of treatment biomarkers for rosacea. However, further studies are needed to verify the functions of the DEGs in physiological and pathological processes.

\section{Material And Methods}

\section{Data collection}

We obtained the gene expression datasets of rosacea patients from the Gene Expression Omnibus (GEO) database (http://www.ncbi. nlm.nih.gov/)[25]. GSE65914 based on platform GPL570 (Affymetrix Human Genome U133 Plus 2.0 Array) was downloaded from the GEO database (Homo sapiens). The dataset of GSE65914 contained 19 patients samples and ten health samples.

\section{Data processing of DEGs}


The GEO2R online analysis tool (https://www.ncbi.nlm. nih.gov/geo/geo2r/), which is an interactive web tool, was used to analyze the gene expression data of microarrays and detected differentially expressed genes (DEGs) between rosacea and health patients[26]. To identify the genes with significant differences in expression, we set the cut-off criteria to filter genes with a fold change (FC) value of $(\| \log F C \mid) \geq 2$ and an adjusted P-value of $<0.05$. We also conducted a distribution to determine whether the samples were suitable for comparison by GEO2R, where the median value indicated that the data were standardized. If not, omit the sample.

\section{Gene ontology (GO) and Kyoto encyclopedia of genes and genomes (KEGG) analysis of DEGs}

Database for Annotation, Visualization, and Integrated Discovery (DAVID, http://david.abcc.ncifcrf.gov/) is considered as the most common functional annotation tool, which was used for gene ontology (GO) functional enrichment analysis and Kyoto Encyclopedia of Genes and Genomes (KEGG) pathway analysis $[27,28,29]$. P-value $<0.01$, false discovery rate $(F D R)<0.01$ and gene counts $>10$ were considered statistically significant.

\section{Integration of Protein-Protein Interaction (PPI) network and hub gene identification}

The Search Tool for the Retrieval of Interacting Genes (STRING) database (http://string-db.org/) is a web tool designed to evaluate the PPI information to reveal proteins' interrelation encoded by selected genes[30,31]. Interaction score of 0.4 was treated as the cut-off criterion. The PPI network was subsequently constructed using Cytoscape software, version 3.8.2 (www.cytoscape.org)[32]. Nodes with higher connectivity tend to be more critical in maintaining the stability of the whole network. CytoHubba, a plugin in Cytoscape, was used to calculate the degree of each protein node. In our study, the top ten genes were identified as hub genes. The function of hub genes was analyzed using DAVID.

\section{TFs Regulatory Network of Hub Genes}

The NetworkAnalyst database (https://www.networkana lyst.ca/) is a web-based tool that contains systemic profiling, meta-analysis, and interpretation of gene expression data[33]. We used the tool to detect the hub genes' transcriptional factors (TFs) and construct the gene-TF regulatory network.

\section{Declarations}

\section{Authors' contributions}


XW, HW and WFD designed and performed the experiments. XW and JJY analyzed and interpreted the data. XW searched the literature and wrote the manuscript. FF revised the manuscript. All the authors reviewed and approved the fnal manuscript

\section{Acknowledgments}

We acknowledge GEO database for providing their platforms and contributors for uploading their meaning datasets.

\section{Funding}

This study was funded by The Foundation of the Chinese Academy of Medical Sciences-Plastic Surgery Hospital (3060120043).

\section{Availability of data and materials}

We obtained the gene expression datasets from the GSE65914 of Gene Expression Omnibus (GEO) database[

\section{Ethics approval and consent to participate}

Not applicable.

\section{Consent for publication}

Not applicable.

\section{Conflict of interest}

The authors declare that they have no competing interests.

\section{References}

1. Zhang H, Zhang M, Wang Y, et al. (2021) Murine models of rosacea: a review. J Cosmet Dermatol.

2. Ebneyamin E, Mansouri P, Rajabi M, et al. The efficacy and safety of permethrin $2.5 \%$ with tea tree oil gel on rosacea treatment: A double-blind, controlled clinical trial. J Cosmet Dermatol. 2020;19:142631. 
3. Two AM, Wu W, Gallo RL, et al. (2015) Rosacea: part I. Introduction, categorization, histology, pathogenesis, and risk factors. J Am Acad Dermatol 72: 749-758; quiz 759-760.

4. Gether L, Overgaard LK, Egeberg A, et al. Incidence and prevalence of rosacea: a systematic review and meta-analysis. Br J Dermatol. 2018;179:282-9.

5. Vera N, Patel NU, Seminario-Vidal L. Rosacea Comorbidities. Dermatol Clin. 2018;36:115-22.

6. Incel Uysal P, Akdogan N, Hayran Y, et al. Rosacea associated with increased risk of generalized anxiety disorder: a case-control study of prevalence and risk of anxiety in patients with rosacea. An Bras Dermatol. 2019;94:704-9.

7. Hayran Y, Şen O, Fırat Oğuz E, et al. (2021) Serum IL-17 levels in patients with rosacea. J Cosmet Dermatol.

8. Amir Ali A, Vender R, Vender R. The Role of IL-17 in Papulopustular Rosacea and Future Directions. J Cutan Med Surg. 2019;23:635-41.

9. Huang Q, Duan L, Qian X, et al. IL-17 Promotes Angiogenic Factors IL-6, IL-8, and Vegf Production via Stat1 in Lung Adenocarcinoma. Sci Rep. 2016;6:36551.

10. Woo YR, Lim JH, Cho DH, et al. (2016) Rosacea: Molecular Mechanisms and Management of a Chronic Cutaneous Inflammatory Condition. Int J Mol Sci 17.

11. Deng $Z$, Lei $X$, Zhang $X$, et al. mTOR signaling promotes stem cell activation via counterbalancing BMP-mediated suppression during hair regeneration. J Mol Cell Biol. 2015;7:62-72.

12. Takeda K, Akira S. TLR signaling pathways. Semin Immunol. 2004;16:3-9.

13. Deng Z, Chen M, Liu Y, et al. A positive feedback loop between mTORC1 and cathelicidin promotes skin inflammation in rosacea. EMBO Mol Med. 2021;13:e13560.

14. Dong R, Zheng S. Interleukin-8: A critical chemokine in biliary atresia. J Gastroenterol Hepatol. 2015;30:970-6.

15. Angiolillo AL, Sgadari C, Taub DD, et al. Human interferon-inducible protein 10 is a potent inhibitor of angiogenesis in vivo. J Exp Med. 1995;182:155-62.

16. Romagnani P, Annunziato F, Lazzeri $E$, et al. Interferon-inducible protein 10 , monokine induced by interferon gamma, and interferon-inducible T-cell alpha chemoattractant are produced by thymic epithelial cells and attract T-cell receptor (TCR) alphabeta + CD8 + single-positive T cells, TCRgammadelta + T cells, and natural killer-type cells in human thymus. Blood. 2001;97:601-7.

17. Sharapova TN, Romanova EA, Sashchenko LP, et al. Tag7-Mts1 Complex Induces Lymphocytes Migration via CCR5 and CXCR3 Receptors. Acta Naturae. 2018;10:115-20.

18. Wilhelm SM, Collier IE, Marmer BL, et al. SV40-transformed human lung fibroblasts secrete a 92-kDa type IV collagenase which is identical to that secreted by normal human macrophages. J Biol Chem. 1989;264:17213-21.

19. Tschesche H, Knäuper V, Krämer $S$, et al. Latent collagenase and gelatinase from human neutrophils and their activation. Matrix Suppl. 1992;1:245-55. 
20. Falay Gur T, Erdemir AV, Gurel MS, et al. The investigation of the relationships of demodex density with inflammatory response and oxidative stress in rosacea. Arch Dermatol Res. 2018;310:759-67.

21. Malik N, Yan H, Moshkovich N, et al. (2019) The transcription factor CBFB suppresses breast cancer through orchestrating translation and transcription. Nat Commun 10: 2071.

22. Ishikawa Y, Kawashima N, Atsuta Y, et al. Prospective evaluation of prognostic impact of KIT mutations on acute myeloid leukemia with RUNX1-RUNX1T1 and CBFB-MYH11. Blood Adv. 2020;4:66-75.

23. Lauper JM, Krause A, Vaughan TL, et al. Spectrum and risk of neoplasia in Werner syndrome: a systematic review. PLoS One. 2013;8:e59709.

24. Kim KH, Roberts CW. Targeting EZH2 in cancer. Nat Med. 2016;22:128-34.

25. Barrett T, Wilhite SE, Ledoux P, et al. NCBI GEO: archive for functional genomics data sets-update. Nucleic Acids Res. 2013;41:D991-5.

26. Dumas J, Gargano MA, Dancik GM. shinyGEO: a web-based application for analyzing gene expression omnibus datasets. Bioinformatics. 2016;32:3679-81.

27. Ashburner M, Ball CA, Blake JA, et al. Gene ontology: tool for the unification of biology. The Gene Ontology Consortium. Nat Genet. 2000;25:25-9.

28. Dennis G Jr, Sherman BT, Hosack DA, et al. DAVID: Database for Annotation, Visualization, and Integrated Discovery. Genome Biol. 2003;4:P3.

29. Kanehisa M, Goto S, Sato Y, et al. KEGG for integration and interpretation of large-scale molecular data sets. Nucleic Acids Res. 2012;40:D109-14.

30. Jensen LJ, Kuhn M, Stark M, et al. STRING 8-a global view on proteins and their functional interactions in 630 organisms. Nucleic Acids Res. 2009;37:D412-6.

31. Szklarczyk D, Franceschini A, Wyder S, et al. STRING v10: protein-protein interaction networks, integrated over the tree of life. Nucleic Acids Res. 2015;43:D447-52.

32. Shannon P, Markiel A, Ozier O, et al. Cytoscape: a software environment for integrated models of biomolecular interaction networks. Genome Res. 2003;13:2498-504.

33. Zhou G, Soufan O, Ewald J, et al. NetworkAnalyst 3.0: a visual analytics platform for comprehensive gene expression profiling and meta-analysis. Nucleic Acids Res. 2019;47:W234-w241.

\section{Figures}




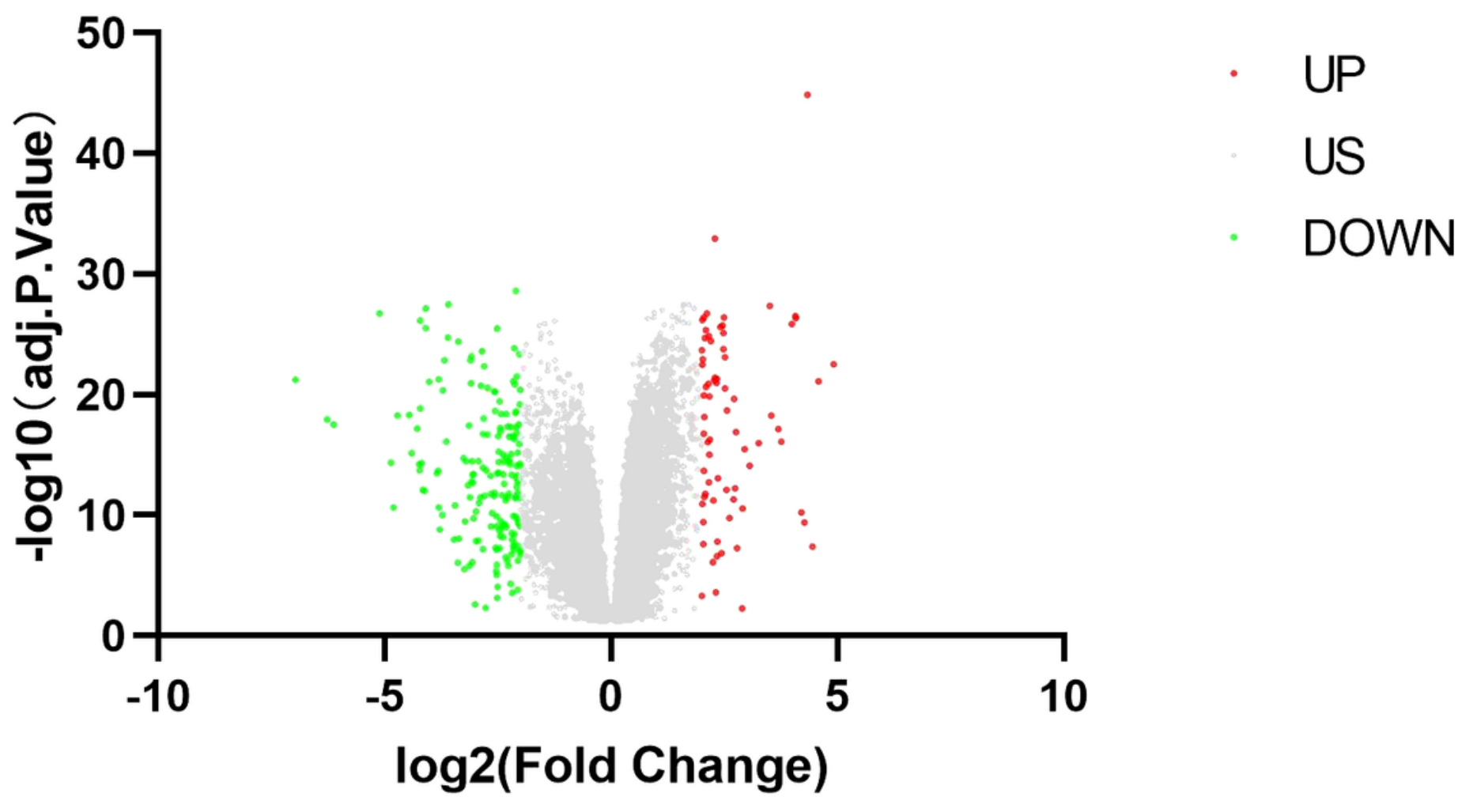

Figure 1

Volcano plot of differentially expressed genes. Genes with changed significantly more than two-fold were selected. Red dot represented up-regulated significant genes and green dot represented down-regulated significant genes. 


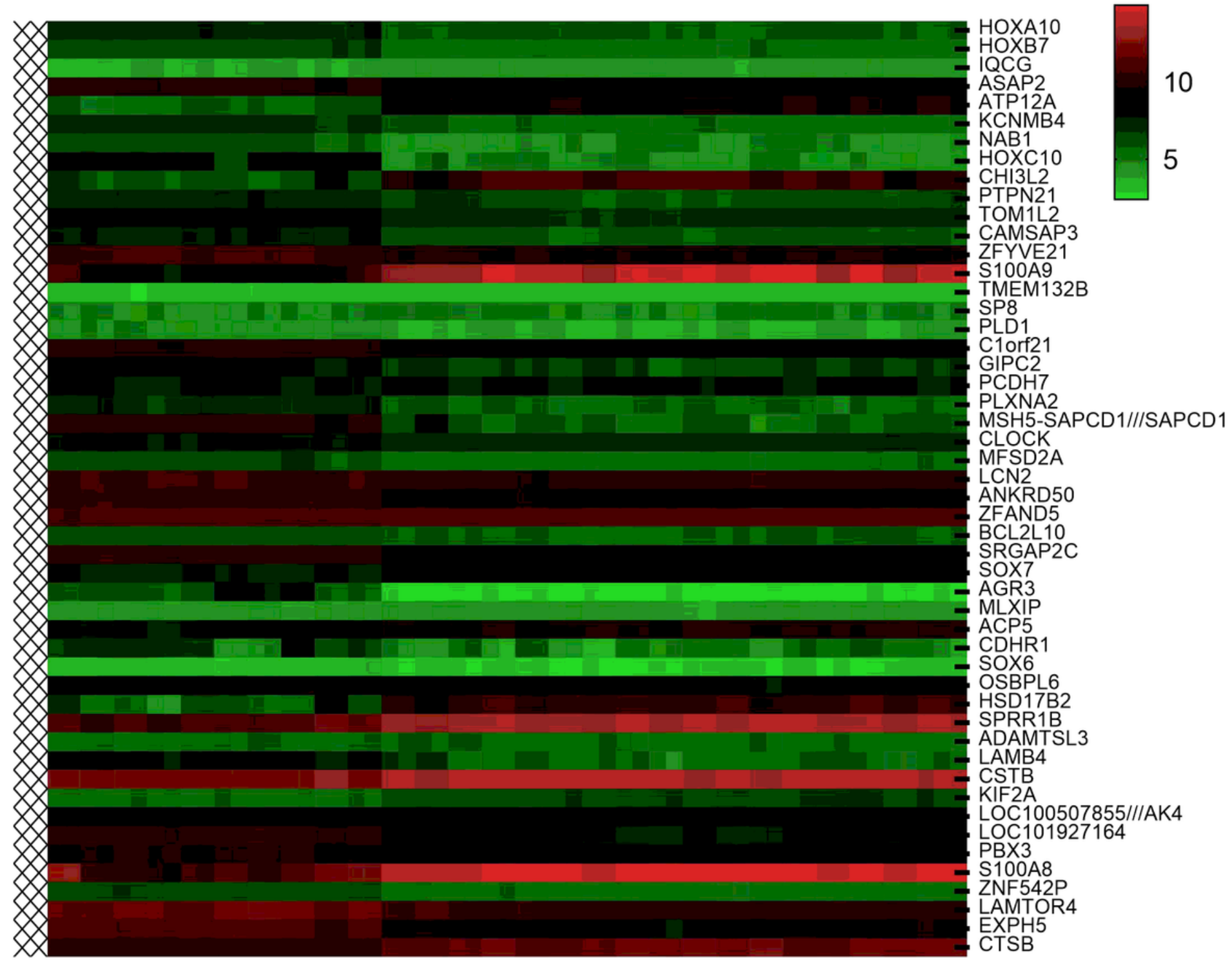

Figure 2

Heatmap of differentially expressed gene expression. The expression profiles greater than the mean are colored in red and those below the mean are colored in green. 


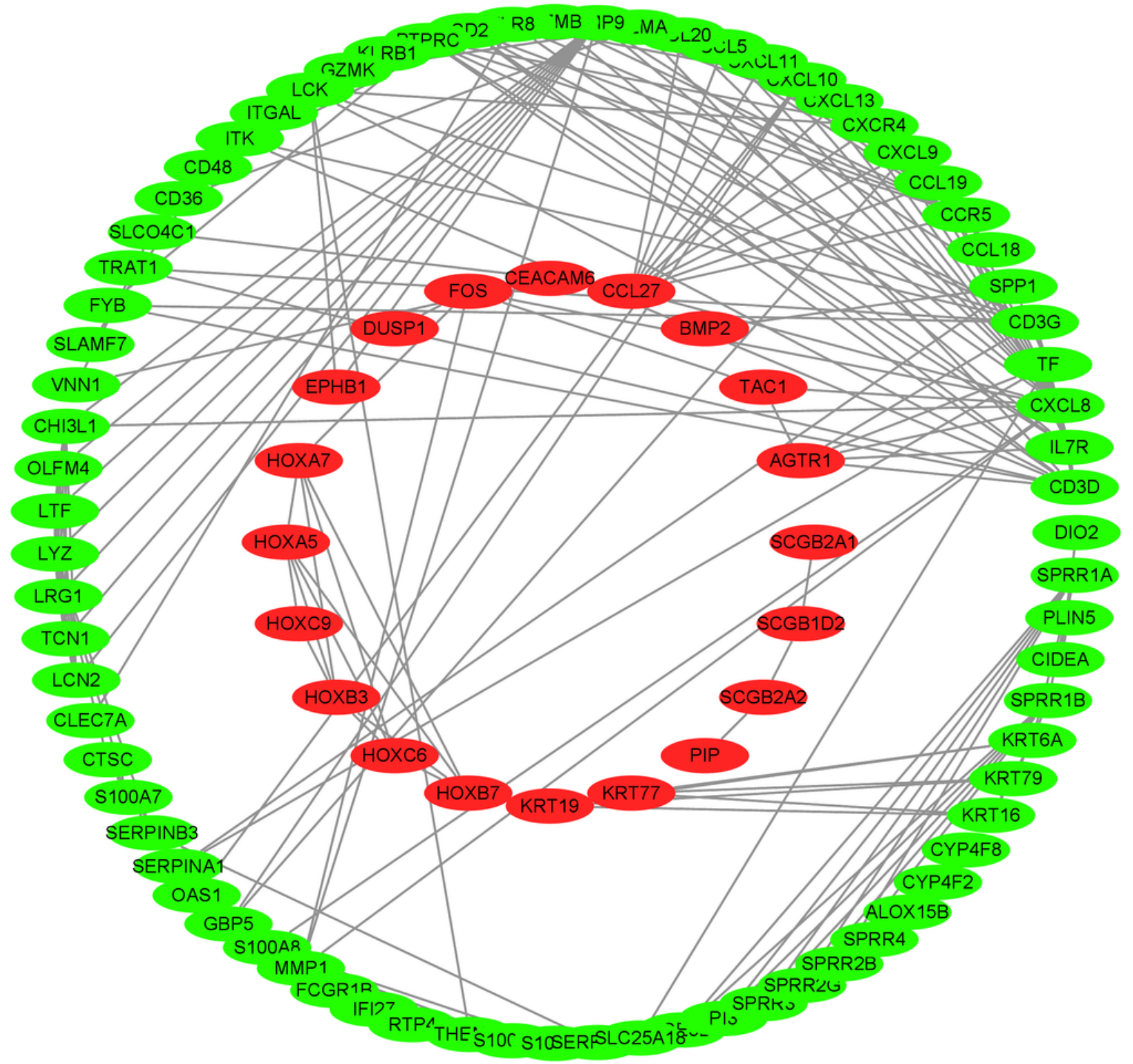

Figure 3

PPI network of DEGs. 


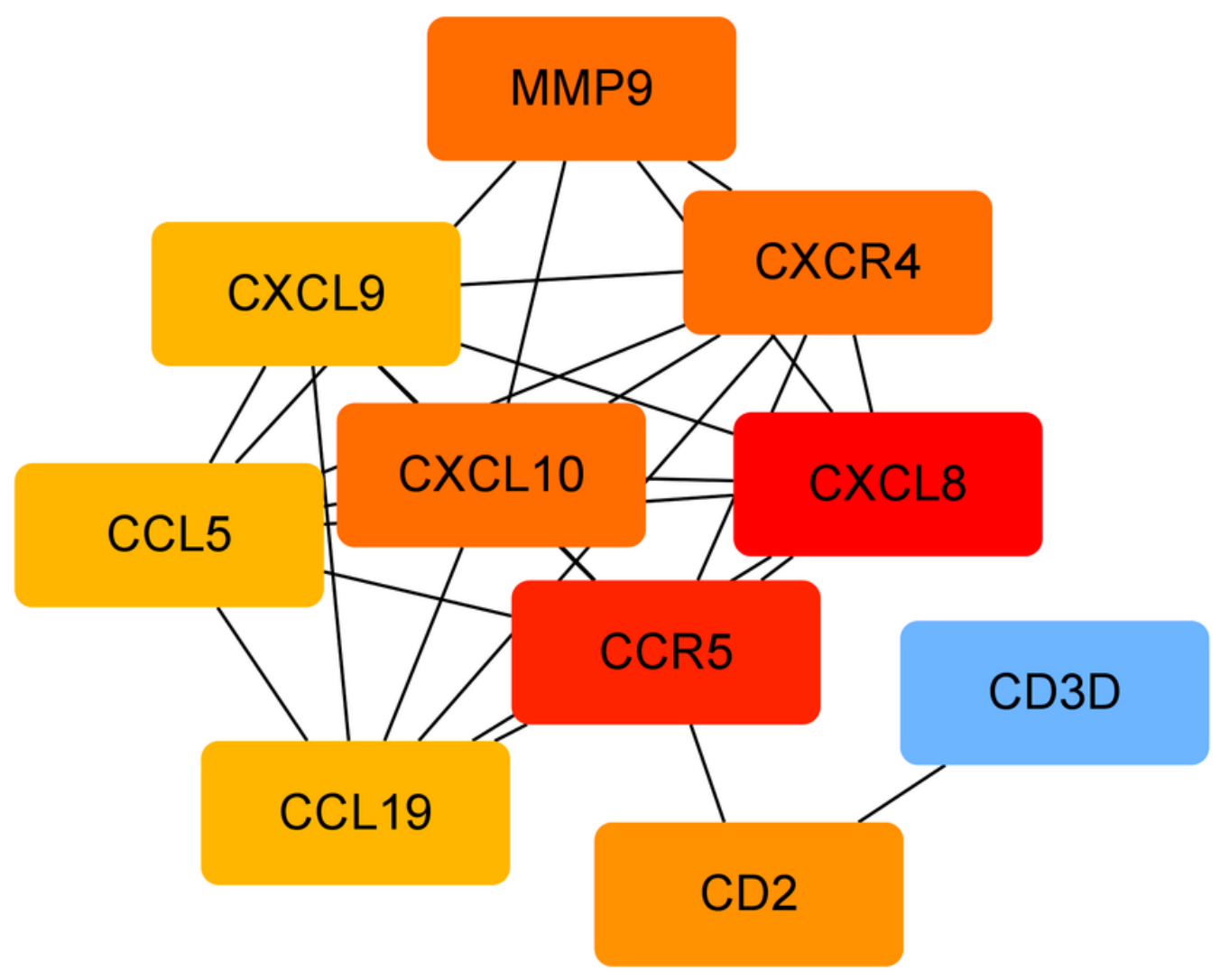

Figure 4

The ten with the highest degrees of connectivity were selected as the hub genes through CytoHubba. 


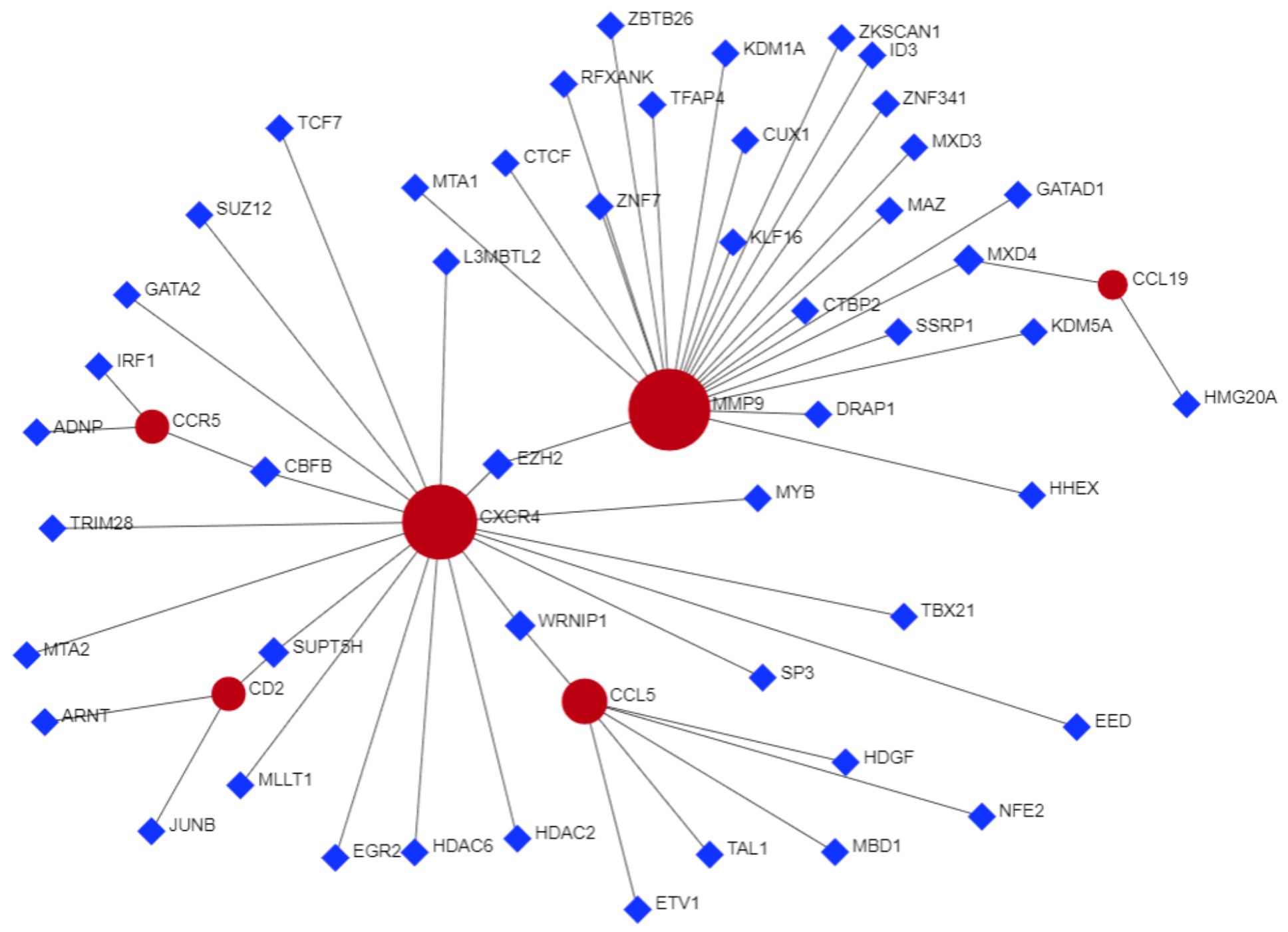

Figure 5

The hub gene-TF regulatory network. Red circle means the hub gene and the blue square means the transcription factor.

\section{Supplementary Files}

This is a list of supplementary files associated with this preprint. Click to download.

- figures1.tif 